\title{
JoVE Methods Collection Highlights: Protein-Protein Interactions
}

\author{
Alastair Barr ${ }^{1}$, Michael Overduin ${ }^{2}$ \\ ${ }^{1}$ School of Life Sciences, University of Westminster ${ }^{2}$ Department of Biochemistry, Faculty of Medicine \& Dentistry, University of Alberta
}

\section{Corresponding Author}

Alastair Barr

A.Barr1@westminster.ac.uk

\section{Date Published}

June 10, 2019

\section{Editorial}

Protein-protein interactions (PPIs) are fundamental to the generation of biological effects. Many are intimately linked with disease, and they are increasingly recognized as offering important targets for drug action ${ }^{1}$. Numerous techniques are available to study these protein interactions, some of which are discussed in this collection or in other JoVE articles. Each technique has its own merits and limitations ${ }^{2}$; however, there is no one perfect technique for all PPIs due to the massive diversity of these interactions which occur in different subcellular compartments, with a wide range of affinities (picomolar to millimolar), and may be only transient in nature.

In vitro kinase assays are an indirect approach for studying PPIs that can be used to analyze how kinases perform their functional role of transferring a phosphate group from ATP to a substrate. Cui et al. ${ }^{3}$ use this technique, coupled with tryptic digest and mass spectrometry, to identify the cyclin-dependent kinase-1-specific phosphorylation sites on a fragment of the human centromere protein $F$ (CENP-F). For functional verification of novel phosphorylation sites, the authors use a binding assay, combining CENP-F containing

\section{Citation}

Barr, A.,Overduin, M. JoVE Methods Collection Highlights: Protein-Protein Interactions. J. Vis. Exp. (148), e59816, doi:10.3791/59816 (2019).
DOI

$10.3791 / 59816$
URL

jove.com/video/59816 a phosphomimetic mutation and karyopherin $\alpha$, a nuclear transport receptor, thus providing cross-validation.

Coimmunoprecipitation is a widely used method for studying PPIs in which an antibody is used to capture a specific target together with any other protein molecules that are associated with it. Zheng and colleagues ${ }^{4}$ apply this technique to study hypoxia-inducible factors (HIFs) that function as heterodimers composed of an oxygen-regulated a subunit and a constitutively expressed $\beta$ subunit also known as ARNT3. An interesting note about their protocol is that the cells are induced and harvested under hypoxic conditions, and endogenous proteins from nuclear fractions are then coimmunoprecipitated. As the authors note, one limitation of the method is that it cannot determine if PPIs are direct or indirect.

Nolan et al. ${ }^{5}$ present a protocol for the measurement of the number of molecules and their brightness in fluorescence microscopy images 'Number and Brightness' (N\&B), that can be applied to detecting protein homo-oligomerization. The technique can be used both in vitro and in vivo and has been applied to accurately quantitate the oligomeric state of mVenus-labelled FKBP12F36V before and after the addition 
of a dimerizing drug. The authors discuss the merits of the technique relative to related technologies and highlight the importance of correcting for bleaching and long-term intensity fluctuations. Confocal microscopes equipped with digital detectors make quantitation easier, but N\&B is also possible with analog detectors.

Affinity purification of multiprotein complexes, together with the identification of their components, has been a widely used technique for understanding the functions of such complexed proteins. In the protocol by Luzarowski et al. ${ }^{6}$, this technique is taken a step further by simultaneously characterizing both PPIs and protein-metabolite interactions (PMIs) in transgenic Arabidopsis plant cells. Tagged nucleoside diphosphate kinases are affinity purified by the tandemaffinity purification (TAP) tag procedure with captured proteins and small molecules subsequently being identified by mass spectrometry. The uniqueness of this protocol is the ability to identify both hydrophobic and hydrophilic ligands; it is a threein-one extraction protocol that enables both PPIs and PMIs to be studied and applies affinity purification to the planting of cells.

The proximity ligation assay has the advantage of being used to detect interactions between endogenous proteins in cells and tissues. Karchugina and Chernoff $^{7}$ use the technique to show the presence of heterodimers of the kinases MST1 and MST2 in human Schwann cells (HSCs) and human embryonic kidney cells (HEK-293). Their article has several valuable pointers on how to perform the technique successfully. Appropriate primary antibodies raised in different species against each interacting protein are required, and since the specificity and sensitivity of the antibodies are key, antibody concentrations should be finely tuned. The authors found glass chamber slides convenient for analyzing multiple cell lines and antibody combinations but noted that it is imperative to remove all of the silicone insert between wells to avoid differences in the confocal distance during microscopy. In addition, various positive and negative controls are used to validate the results.

Nuclear magnetic resonance (NMR) spectroscopy is a sensitive assay that can be used to provide atomiclevel resolution and quantitative information about protein interactions and protein-ligand interactions. Winkelaar and colleagues $^{8}$ use the technique to study the interaction of two proteins (vimentin and envoplakin) found in desmosomes, cell structures involved in cell-to-cell adhesion that are typically found in tissues that experience intense mechanical stress. The authors use a ${ }^{15} \mathrm{~N}$-labelled vimentin domain and acquire a 2D spectrum using heteronuclear single quantum correlation (HSQC). In the presence of a domain from envoplakin, extensive line broadening and peak disappearance are observed, consistent with protein interaction. The authors further probe the basis of the interaction using mutagenesis and microscale thermophoresis (MST) to provide quantitative information.

Small-angle X-ray scattering (SAXS) is a technique that can be used to provide information on the size and conformations of a macromolecule in solution and generate a molecular envelope of large, multidomain or protein complexes at low resolution. In the protocol by Mrozowich et al. ${ }^{9}$, the technique is used to generate a low-resolution structure of nidogen-1 and laminin $\mathrm{y}-1$ in complex. The technique may be of interest to researchers studying PPIs in large proteins with multiple globular domains that are too big for NMR spectroscopy and are challenging to crystallize for X-ray crystallography.

It has been estimated there are some 650,000 interactions in the human interactome ${ }^{10}$ _enough to keep researchers 
in the field busy for some time to come. A recent development, made possible by advances in microscopy, mass spectrometry, and machine learning, is spatial proteomics that may provide great insight in this area in the future ${ }^{11}$. This collection of PPI methods will help researchers tackle this important challenge while avoiding potential pitfalls.

\section{Disclosures}

The authors have nothing to disclose.

\section{Acknowledgments}

The authors acknowledge NSERC RGPIN-2018-04994, Campus Alberta Innovation Program (RCP-12-002C), and Alberta Prion Research Institute/Alberta Innovates Bio Solutions (201600018) grants awarded to M.O.

\section{References}

1. Jin, L., Wang, W., Fang, G. Targeting protein-protein interaction by small molecules. Annual Review of Pharmacology and Toxicology. 54, 435-456 (2014).

2. Titeca, K., Lemmens, I., Tavernier, J., Eyckerman, S. Discovering cellular protein-protein interactions: Technological strategies and opportunities. Mass Spectrometry Reviews. 38 (1), 79-111 (2019).

3. Cui, H., Loftus, K. M., Noell, C. R., Solmaz, S. R. Identification of Cyclin-dependent Kinase 1 Specific Phosphorylation Sites by an In Vitro Kinase Assay. Journal of Visualized Experiments. (135), e57674 (2018).

4. Zheng, X. et al. Co-immunoprecipitation Assay Using Endogenous Nuclear Proteins from Cells Cultured
Under Hypoxic Conditions. Journal of Visualized Experiments. (138), e57836 (2018).

5. Nolan, R. et al. Calibration-free In Vitro Quantification of Protein Homo-oligomerization Using Commercial Instrumentation and Free, Open Source Brightness Analysis Software. Journal of Visualized Experiments. (137), e58157 (2018).

6. Luzarowski, M., Wojciechowska, I., Skirycz, A. 2 in 1: One-step Affinity Purification for the Parallel Analysis of Protein-Protein and Protein-Metabolite Complexes. Journal of Visualized Experiments. (138), e57720 (2018).

7. Karchugina, S., Chernoff, J. Detection of Heterodimerization of Protein Isoforms Using an in Situ Proximity Ligation Assay. Journal of Visualized Experiments. (140), e57755 (2018).

8. Winkelaar, G., Trieber, C., Kumar, J., Overduin, M. Measuring Interactions of Globular and Filamentous Proteins by Nuclear Magnetic Resonance Spectroscopy (NMR) and Microscale Thermophoresis (MST). Journal of Visualized Experiments. (141), e58537 (2018).

9. Mrozowich, T., McLennan, S., Overduin, M., Patel, T. R. Structural Studies of Macromolecules in Solution using Small Angle X-Ray Scattering. Journal of Visualized Experiments. (141), e58538 (2018).

10. Stumpf, M. P. et al. Estimating the size of the human interactome. Proceedings of the National Academy of Sciences of the United States of America. 105 (19), 6959-6964 (2008).

11. Lundberg, E., Borner, G. H. H. Spatial proteomics: a powerful discovery tool for cell biology. Nature Reviews Molecular Cell Biology. (2019). 